\section{LOS CINCO SEXOS, O CÓMO ESTABLECEMOS FRONTERAS CATEGORIALES MORALMENTE RELEVANTES EN UN MUNDO DIFUSO Y CONTINUO}

\author{
Antoni Gomila \\ Dep. Psicología. Univ. Illes Balears \\ toni.gomila@uib.es
}

\begin{abstract}
Cómo citar este artículo/ Citation: Gomila, A. (2013). "Los cinco sexos, o cómo establecemos fronteras categoriales moralmente relevantes en un mundo difuso y continuo". Arbor, 189 (762): a050. doi: http://dx.doi.org/10.3989/ arbor.2013.762n4001
\end{abstract}

Recibido: 13 julio 2012. Aceptado: 6 junio 2013.

RESUMEN: Generalmente se considera que el realismo moral exige un mundo bien determinado, para que las valoraciones también puedan estarlo. Desde este punto de vista, los dilemas morales genuinos, donde no pueda establecerse la opción moralmente correcta porque haya razones igualmente poderosas para ambas alternativas, constituyen un desafío al realismo moral. En este trabajo argumento en contra de este tipo de ataque al realismo moral. Partiendo del carácter continuo y gradual de los criterios constitutivos de nuestros conceptos, muestro cómo cabe esperar precisamente casos límite, en los que aparentemente está indeterminada la verdad. Que existan fronteras conceptuales borrosas no significa que no puedan ser cruzadas (que no sean útiles, que no capten diferencias o aspectos relevantes desde el punto de vista de la estructura causal de la realidad y su inteligibilidad), al contrario: sirven para establecer diferencias moralmente relevantes. De hecho, la posibilidad de una comunidad con prácticas conceptuales compartidas depende de la existencia de los casos paradigmáticos, prototípicos, centrales, de ejemplificación de un concepto, y de valoración moral.

PALABRAS CLAVE: realismo moral; indeterminación semántica; casos paradigmáticos.

\section{THE FIVE SEXES, OR HOW WE ESTABLISH MORALLY RELEVANT CATEGORIES IN A DIFFUSE AND CONTINUOUS WORLD}

Copyright: (c) 2013 CSIC. Este es un artículo de acceso abierto distribuido bajo los términos de la licencia Creative Commons Attribution-Non Commercial (by-nc) Spain 3.0.

ABSTRACT: It is generally assumed that moral realism requires a determined world, so that moral judgments can also be determined. From this point of view, genuine moral dilemmas, where two alternative judgments are equally well-grounded, would be a counter-example for moral realism. In this paper I argue against such a challenge to moral realism. Starting from the continuous and gradualist character of the constitutive criteria of our concepts, I show how indeterminacies and limit cases are to be expected where it can be impossible to determine the truth value of a proposition. The existence of fuzzy conceptual boundaries does not mean they cannot be crossed (that they are not useful, that they do not capture difference or relevant features from the point of view of the causal structure of reality and its intelligibility), quite the opposite: they serve to establish morally relevant differences. The very possibility of a community with common conceptual practices depends upon the existence of paradigmatic, central cases that exemplify a concept meaning or a moral valuation.

KEYWORDS: moral realism; semantic indeterminations; paradigmatic cases. 


\section{BIOÉTICA: PROBLEMAS DE FRONTERAS}

Al dedicar este volumen a una discusión sobre las fronteras biológicas y los límites legales, sus promotores han focalizado nuestra atención en una cuestión fundamental a la que se enfrenta la Bioética: el de la justificación de las propuestas normativas que se plantean en un determinado ámbito. Un modo de justificarlas, a primera vista natural, consistiría en basarlas en distinciones propiamente biológicas, o en general, naturalistas: cosas diferentes deben ser tratadas - juzgadas - de modo distinto. Un modo de enfrentarse a este tema, por tanto, consiste en plantear si las decisiones normativas deben basarse única o exclusivamente en consideraciones naturalistas (acerca de cómo son las cosas), o bien si hay otras fuentes de normatividad que deban ser tenidas en cuenta (religiosas o trascendentales, por ejemplo).

Una segunda aproximación posible consiste en plantear si, dadas o reconocidas ciertas diferencias naturales, tales diferencias tienen relevancia moral o normativa en general, puesto que claramente no todas las diferencias "fácticas" tienen relevancia normativa. Así, por ejemplo, actualmente rechazamos que de las diferencias entre hombres y mujeres, diferencias étnicas o ideológicas, se sigan diferencias en derechos o en estatus legal (y mucho menos del color del pelo, o del número de pie). Es desde este punto de vista que surge la vieja advertencia humeana de la diferencia lógica entre el ámbito descriptivo y el normativo, y la necesidad de no derivar un "debe" de un "es" alegremente. Aunque se ha sostenido, y suele darse por supuesto que ese salto es siempre lógicamente incorrecto (la llamada "falacia naturalista"), la advertencia de Hume no iba tan lejos, y solo alertaba del salto lógico y reclamaba explicitar la justificación de tal salto, si tal cosa es posible; hay quien cree que sí es posible tal justificación: derivar un "debe" de un "es" puede verse como un entimema, un argumento donde la premisa normativa, que establece la relevancia normativa de esa diferencia natural, está implícita. La derivación es falaz, por tanto, solamente si esa premisa implícita no puede ser explicitada y justificada (Rabossi, 2002). En el caso de la justificación del aborto, por ejemplo, se puede defender que el aborto está permitido en las primeras semanas de gestación (conclusión deóntica) a partir de la constatación fáctica de que no se ha constituido todavía una persona, en el supuesto implícito de que las restricciones normativas se aplican solo a las personas y no a los blastocitos. $O$ el rechazo a las corridas de toros (debe) por el dolor que experimentan estos mamíferos en el curso de ese espectáculo (es), presupone la premisa de que está mal causar dolor para divertirse. La derivación normativa puede justificarse si justificamos en primer lugar la premisa normativa implícita.
Nótese que en estos casos puede ser objeto de discusión tanto la consideración descriptiva, como la premisa normativa que justifica la inferencia normativa. En efecto, se puede discutir si realmente los toros pueden experimentar dolor; por ejemplo, a través de una caracterización muy restrictiva e intelectualizada de la capacidad de sentir dolor, que pueda dejar fuera de su extensión a todos los animales (a costa de dejar fuera también a algunos humanos), estrategia que sigue, por ejemplo, Carruthers (2005). O bien se puede discutir si ciertamente está mal causar dolor para divertirse, o si puede estar justificado cuando esa diversión tiene un carácter ritual, por ejemplo. En cualquier caso, quisiera advertir a quienes suelen aceptar como axiomática la falacia naturalista que deberían, para ser coherentes, eliminar las consideraciones de hecho (por ejemplo, sobre si los animales pueden o no sentir dolor) en su reflexión moral, y asumir, al modo de Moore, la existencia de una facultad de "intuición" como origen del conocimiento moral.

Pero la propuesta de este volumen puede verse también desde el punto de vista más preciso y concreto: aun aceptando una posición naturalista y realista sobre las normas, se plantea la dificultad de que las fronteras "naturales" pueden resultar insuficientes para establecer diferencias normativas, por la posibilidad - meramente hipotética, quizá, o derivada de las nuevas capacidades técnicas en el campo de la biomedicina - de encontrarse con casos límite, casos cuya mera descripción no resulta obvia, y que, por ende, resulta todavía menos clara su calificación normativa. Este es, al menos, el problema que trataré de afrontar en este trabajo, a partir del reconocimiento del fenómeno de la borrosidad y tipicidad de nuestros conceptos. Es decir, trataré de situar el fenómeno de los casos límite, que nos piden considerar los editores, como un caso particular del fenómeno general de la vaguedad e indeterminación de las condiciones de uso de nuestros conceptos. Según trataré de mostrar, este aspecto de nuestras capacidades cognitivas no puede atribuirse exclusivamente a la falta de precisión de nuestro sistema conceptual "de sentido común" (frente a la esperable precisión del conocimiento científico, por ejemplo), o a una limitación de nuestro lenguaje (su falta de precisión, reformable con ayuda de la lógica, por ejemplo), sino que tiene que ver también con el carácter dinámico y continuo de nuestro mundo.

Tras describir la naturaleza de los casos límite y su relevancia normativa (sección 2), e ilustrar el modo habitual de enfrentarse a tales casos con el ejemplo del sexo, como un intento paradigmático de evitar tal borrosidad (sección 3), ofreceré una breve presentación del fenómeno de la borrosidad conceptual y la indeterminación semántica en el marco de las ciencias cognitivas contemporáneas (sección 4). Situaré a 
continuación, y trataré de ilustrar, en la siguiente sección, las fuentes de la indeterminación semántica en el caso de los conceptos morales, y discutiré su relevancia respecto a la cuestión del realismo moral (sección 5). Por supuesto, al señalar la ubicuidad de la borrosidad conceptual y la indeterminación semántica no pretendo cambiar el modo en que se desarrolla la Bioética (tratando de perfilar las fronteras biológicas relevantes y determinar los límites normativos válidos), sino más bien calmar cierta ansiedad metafísica: la que alienta tras el supuesto, compartido tanto por los realistas deterministas como por los anti-realistas, de que solo cabe hablar de realismo (moral) en un mundo perfectamente determinado y definido.

\section{BORROSIDAD, CASOS LÍMITE Y DILEMAS MORALES}

La existencia de casos límite, de casos borrosos, donde no es fácil establecer cuándo deja de ser aplicable un concepto y cuándo se ejemplifica mejor otro, o en que una misma situación puede ser conceptualizada de modos distintos, no es exclusiva del ámbito moral, sino que es una característica general de nuestro sistema semántico-cognitivo (Kamp \& Partee, 1995). Si bien tradicionalmente este problema se reconoció solamente en relación con los predicados unidimensionales, como calvo o alto, que remiten a propiedades que varían de manera continua (pelo a pelo, centímetro a centímetro), pero para los que no es posible determinar el punto exacto de transición (cuándo uno empieza a ser calvo, o a partir de qué altura alguien es alto), actualmente se da por supuesto que todos nuestros predicados presentan una estructura gradual (una aplicación de más a menos, un gradiente de tipicidad) que se convierte en borrosidad cuando nos acercamos a los límites de la clase de referencia. La idea, para ser más precisos, no es que haya predicados borrosos, sino casos para los que puede no ser claro, o determinado, si satisfacen o no un predicado dado. No es que "calvo" sea un predicado borroso, sino que aplicado a mí, por ejemplo, puede convertirse en un caso borroso, límite, dando lugar a una proposición indeterminada (al menos, de momento; llegará un día en que "AG es calvo" será un proposición perfectamente determinada y verdadera). Como veremos, esta propiedad resulta de la estructura prototípica de nuestros conceptos, que determina que ciertos casos sean más típicos de este concepto que otros (un canario ejemplifica mejor "pájaro" que un pingüino; una rosa ejemplica mejor "flor" que una coliflor), hasta un punto de borrosidad fronteriza, en que ya no está claro si un caso satisface el concepto o no (¿un tomate es una fruta?).

Este carácter continuo, gradual, de los criterios constitutivos de nuestros conceptos posibilita el surgimiento de los casos límite, casos en los que aparentemente está indeterminada la verdad, o donde no parece posible escoger entre varias descripciones de la misma situación. En el caso de los predicados unidimensionales como "calvo", consistirá en aquellos casos en donde no hay evidencia para establecer si " $x$ es calvo", para elegir entre esta afirmación y " $x$ no es calvo". En el caso de los predicados morales podemos encontrarnos con problemas análogos, como el de cuánto cerebro hace falta para que exista una persona (con casos como el de los anencefálicos, o dicefálicos, pero también casos hipotéticos, o experimentos mentales, que nos permiten imaginar la indeterminación), o cuánta actividad cerebral debe darse para poder seguir afirmando que alguien está vivo (la muerte cerebral como criterio de muerte). La borrosidad de la situación genera la indeterminación semántica en tales casos: son los casos límite (Shafer-Landau, 1995).

Los casos límite, por tanto, sirven para plantear dilemas éticos (Sorensen, 1991): la posibilidad de los dilemas éticos depende de este margen de ambigüedad acerca de la caracterización adecuada del caso límite, compatible con consecuencias normativas distintas, en función de la relevancia de principios distintos, según la caracterización del caso que sea más acertada. Así, por ejemplo, ante el caso de una persona mayor con riesgo de gangrena si no es intervenida rápidamente, pero de la que hay que obtener consentimiento informado previo, el médico puede encontrarse ante obligaciones en conflicto, en función del grado de gravedad y de incapacidad (y ese grado puede variar de manera continua). Es la posibilidad de grados lo que lleva al dilema, al menos en el plano hipotético, pues podemos "modificar" convenientemente el caso para que el "peso" de cada una de las consideraciones morales sea el mismo, y por tanto, aparezca el dilema. El médico puede estar, al mismo tiempo, obligado a intervenir (sin esperar el consentimiento), y obligado a no intervenir (hasta disponer del consentimiento).

La repercusión que este tipo de situaciones supone a nivel normativo puede verse como la necesidad, y la dificultad, de "imponer" límites regulativos estrictos donde parece no haberlos en el plano biológico o natural. O bien, la necesidad de modificar el modo establecido de fijar las distinciones naturales, introduciendo nuevos elementos descriptivos, que puedan tener relevancia normativa. En efecto, podemos encontrarnos una continuidad de situaciones a primera vista análogas, para las que nos parece que debería haber un tratamiento normativo diferenciado, pero cuya justificación de tales diferencias no resulta obvia. Desde este punto de vista, creo que adquiere pleno sentido la afirmación de que el dominio de la Bioética es el de las fronteras. 0 más precisamente, el de determinar en qué lado de la frontera (normativa) cabe situar adecuadamente un caso particular, que desde el punto de vista biológico parece borroso. Bien sea el caso de establecer cuándo alguien está muerto, y si 
la muerte cerebral constituye el limes decisivo para el cambio de estado, así como determinar cuándo se ha producido; bien sea el de proponer un criterio para la existencia de vida humana, en base al cual podamos juzgar casos particulares,... los problemas de la Bioética parecen tener que ver con establecer distinciones dentro de un continuo, y fijar la ubicación de los casos dentro de ese continuo ${ }^{1}$. Por eso no es de extrañar que una de las formas argumentativas más recurridas en este ámbito sea el de la "pendiente deslizante": a partir de aceptar un punto de partida compartido, se argumenta que otros casos son como el de ese punto de partida en algún aspecto relevante, y por lo tanto, deben tratarse igual. Resistirse a tal "pendiente deslizante" requiere poder establecer una frontera, una diferencia fáctica, normativamente relevante.

Una indicación de la relevancia de este modo de considerar las cosas se obtiene al observar que los mismos problemas de indeterminación se plantean al nivel de los predicados normativos. Es decir, que sería erróneo pensar que es únicamente en el plano biológico, o natural, donde ocurren los casos límite, donde tenemos fronteras borrosas, mientras que en el normativo disponemos de conceptos bien afilados y determinados. Al contrario, también en este ámbito se plantean casos donde puede haber razones igual de poderosas para calificar los casos que se plantean de modos distintos. Es lo que sería de esperar si la borrosidad y vaguedad semántica es una característica general de nuestro sistema conceptual, y no una molesta rémora que debe y puede ser eliminada con un poco de esfuerzo de precisión y definición.

Desde este punto de vista, podría decirse que, al enfrentarse a los casos límite, y a los dilemas morales que tales situaciones pueden plantearnos, la Bioética suele adoptar una vía de búsqueda de mayor precisión normativa y casuística: de aclarar las pequeñas diferencias que pueden resultar relevantes desde un punto de vista normativo, de introducir nuevas distinciones y clasificaciones no tenidas en cuenta en la descripción inicial del caso, que puedan inclinar la decisión en un sentido o en otro. Sin cuestionar el valor que tiene este modo de proceder, creo que también puede ser útil reflexionar sobre el por qué se plantean las cosas en estos términos en primer lugar; en particular, por qué son posibles tales casos límite $-y$ el por qué esas resoluciones pueden desembocar en nuevos casos límite, como suele ocurrir-. Esto es lo que me gustaría proponer en este trabajo, adoptando una perspectiva semántico-cognitiva, que sitúe este fenómeno de los casos límites morales en el contexto más amplio de la vaguedad de nuestros predicados.

Planteada así la cuestión, sería posible sugerir, en la línea del primer tipo de problemática planteada, que en realidad no es acertado buscar la justificación de las fronteras normativas en fronteras biológicas, puesto que estas últimas no están bien definidas, o su establecimiento depende a la vez de valores (epistémicos, sociales), por lo que las decisiones normativas no pueden tener otra base que el acuerdo, la convención; o en una versión menos social, la pura decisión, la preferencia emocional, la arbitrariedad de la voluntad. Se trataría de adoptar alguna variante de constructivismo o decisionismo normativo, o de expresivismo, que asuma un antirealismo en el ámbito metaético. Volveremos sobre esta cuestión de las implicaciones del reconocimiento de la vaguedad conceptual al final. La opción predominante, no obstante, sigue siendo la realista, que trata de buscar o establecer una nueva distinción, una nueva frontera, en el plano biológico, y basar en ella la diferencia moral, su relevancia normativa, y por tanto, la posibilidad de parar un argumento de pendiente deslizante (o en negar su relevancia). Y esta aproximación dominante concibe esta tarea en términos de razones, de corrección, de tener en cuenta cómo son las cosas. Un ejemplo notorio, aunque difícil de plantear sin prejuzgarlo, es de nuevo el del aborto. La posición antiabortista parte de que matar a un persona está mal; y sostiene que no hay ninguna diferencia relevante entre un bebé y un blastocito, por lo que si alguien acepta que se puede matar a un blastocito también debe aceptar, para ser coherente y racional, que se puede matar a un bebé (por pendiente deslizante). Una línea de defensa de la aceptabilidad ética y jurídica del aborto consiste en tratar de establecer una diferencia constitutiva entre los blastocitos y los bebés, que bloquee la pendiente deslizante. Si se prefiere un caso de menor carga emocional, piénsese en el papel que se concede en el ámbito jurídico al grado de intencionalidad de una acción para calificarla penalmente, $\mathrm{o}$ al sentido de las atenuantes o las eximentes en el ámbito jurisdiccional: diferencias en el plano descriptivo de la acción modifican la consideración normativa del caso.

Ilustraré a continuación la naturaleza y las limitaciones de esta estrategia con un caso paradigmático desde el punto de vista biológico, pero que presenta también cierta relevancia bioética, como es el de los sexos. Este caso nos mostrará que la cuestión de los casos borrosos no se resuelve simplemente enriqueciendo nuestras taxonomías con nuevos conceptos y clases, pues el problema se reproduce en un segundo nivel.

\section{LOS CINCO SEXOS}

En 1993, Anne Fausto-Sterling publicó un artículo en la revista The Sciences, titulado "The five sexes", en el que defendía que el sistema universal de distinguir dos sexos no es adecuado para abarcar todo el espectro de la sexualidad humana. Exponía como base para tal conclusión su estudio de la prevalencia de casos 
de los que no es obvio afirmar si se trata de un varón o de una mujer, por la combinación diversa de características masculinas y femeninas que puede darse. Por ello, sugería modificar la clasificación dicotómica tradicional universal (que el estructuralismo de LeviStrauss tomó como piedra angular de su concepción de lo humano), introduciendo nuevas categorías, además de varón y mujer:

- "herms", para los verdaderos hermafroditas, personas con testículos y ovarios;

- "merms", para los pseudohermafroditas masculinos, personas nacidas con testículos y que desarrollaron algunos aspectos de la genitalidad femenina; $y$

- "ferms", para los pseudohermafroditas femeninos, que tienen ovarios, en combinación con algunos aspectos de la genitalidad masculina.

No hace falta detenernos en este punto en la relevancia normativa de la propuesta, en la que podemos estar de acuerdo sin problema: lo que motivaba a Fausto-Sterling era reivindicar el reconocimiento de unas realidades humanas que eran forzadas a encajar en las categorías sociales dicotómicas establecidas, generando graves daños en la vida de I@s afectad@s, al ser sometid@s a cirugía temprana e irreversible. Aun ahora que tales prácticas están en remisión, las personas que no encajan en los géneros reconocidos puede seguir padeciendo discriminaciones y acoso (Amnistía Internacional, 2006).

Lo que nos interesa del caso es hacer notar, en primer lugar, lo que genera el "problema": la existencia de diferentes criterios para establecer cuál es el sexo de alguien, y la aparición de casos en que tales criterios no coinciden. El criterio genotípico, cromosómico, para ser un varón consiste en tener un cromosoma $X$ y uno $Y$, mientras que las mujeres tienen un par de cromosomas X. A nivel fenotípico puede haber varios criterios: a nivel anatómico-fisiológico, varones y mujeres difieren en sus genitales y en sus gónadas; a nivel hormonal, las diferencias sexuales se acaban manifestando, tras la pubertad, en diferentes características sexuales secundarias (pelo en la cara, musculatura, voz más grave,... en los varones; mamas en las mujeres). Pues bien, una mutación genética, relativamente frecuente entre los esquimales Yupik, por ejemplo, puede dar lugar a la Hiperplasia Adrenal Congénita, dominante cuando se hereda de ambos padres, que consiste en contar con dos cromosomas $X$, unos genitales externos masculinos, y órganos reproductivos internos femeninos potencialmente fértiles. ¿Cuál es el sexo de tales personas?

Por supuesto, el problema real que se plantea en este tipo de casos, y de ahí el entrecomillado anterior, no es puramente terminológico, de cómo denominar- los, sino el práctico de cómo tratarlos: aceptando la variabilidad de la realidad, o negándola y sometiéndola al corsé de las posibilidades reconocidas, varón y mujer, con sus implicaciones legales, sociales, de género, que esa asignación comporta. El artículo de Fausto-Sterling, así como el movimiento de los "intersexuales", liderado por Cheryl Chase (Intersex Society of North America), han abogado por modificar la pauta médica establecida de someter a cirugía "normalizadora" a los bebés que presentaban algún caso de genitalidad mixta en el momento del nacimiento, para "desambiguarlos sexualmente", en la creencia de que la identidad sexual es completamente maleable en los primeros 18 meses. Frente a esta pauta establecida, se han propuesto nuevos criterios de no urgencia y no irreversibilidad respecto a la cirugía temprana, dejando que cada persona encuentre su identidad sexual en la pubertad (para lo cual puede ser necesario apoyo psicológico, y quizá a partir de ese momento, cirugía irreversible o tratamiento hormonal complementario).

Aunque los objetivos de tal reivindicación me parecen adecuados (el caso de Chase es el de alguien que fue sometida a cliteridectomía con la intención de "hacerlo un hombre"), creo que la propuesta de introducir una nueva nomenclatura, una reforma taxonómica de los sexos, ilustra bien esta inquietud ante los casos borrosos, los límites mal definidos, y la reacción típica de tratar de convertirlos en precisos y bien delimitados, mediante una ampliación de la clasificación. Ciertamente, si se adoptara el sistema clasificatorio propuesto por Fausto-Sterling, algunos de los casos que resultaban indeterminados, dejarían de serlo. Pero me gustaría hacer notar dos cosas: que las nuevas categorías son construidas en base a las de varón y mujer, por lo que se trata en realidad de un intento de precisificación de segundo orden, que presupone la determinación del primero; y ello hasta el punto que el efecto práctico de la propuesta radica en no precipitar la desambiguación sexual, en permitir que pueda ser cuestión de decisión individual, de ajuste psicológico, pero dándose por supuesto que la intersexualidad biológica va a acabar resolviéndose en uno de los dos sexos-géneros; y segundo, que por ello mismo, su propuesta es susceptible de nuevas indeterminaciones, en las nuevas fronteras que pretende fijar (así, ¿cómo cabría clasificar un caso de trisomía XXY, o XYY? ¿o unos genitales a medio camino entre una vulva y un pene? etc. $)^{2}$.

En la medida en que la frecuencia de tales casos sea baja, no obstante, y los estudios realizados hablan de una prevalencia del $1,7 \%$, aunque con gran variabilidad poblacional, y no aparezca un tercer género, no se ve la ventaja de modificar el sistema clasificatorio tradicional para intentar ser más precisos, al menos desde el punto de vista de la economía cognitiva. De hecho, la propia Intersex Society of North America re- 
comienda asignar provisionalmente cada niño a un género, masculino o femenino, en función del pronóstico de cómo va a sentirse cuando crezca, en lugar de reivindicar la introducción de un "tercer sexo/ género". Según se afirma, "el intersexo no es, ni será nunca, una categoría biológica discreta, más de lo que hombre o mujer lo son, y porque tal asignación traumatizaría innecesariamente al infante" (www.isna. org). (De todos modos, resulta fascinante imaginar la posibilidad de una sociedad que estableciera más de dos categorías de sexo-género, donde las relaciones no fueran solo homo y heterosexuales, etc.).

\section{CONCEPTOS, TIPICIDAD Y BORROSIDAD}

La discusión del caso anterior nos lleva a la conclusión de que la borrosidad de nuestros conceptos no es una característica que pueda ser eliminada o superada introduciendo mayor precisión, pues en este nuevo nivel puede aparecer de nuevo la borrosidad. Ciertamente, el programa logicista, desde Frege, sueña con un esquema conceptual perfectamente bien definido y determinado, en un mundo bien determinado. Pero esta visión está actualmente en retroceso. La idea de que nuestros conceptos no contienen "esencias ocultas", que captan las propiedades ónticas de la realidad, sino que se constituyen en prácticas simbólicas sociales, basadas en semejanzas de familia, constituye el punto básico de las Investigaciones Filosóficas de Wittgenstein. A partir de los años 70, este planteamiento filosófico dio lugar a un programa de investigación cognitiva sobre la naturaleza de las categorías y los conceptos, impulsado principalmente por Eleonor Rosch (Rosch \& Mervis, 1975).

Su contribución principal consistió en mostrar el fenómeno de la métrica gradual de pertenencia a la extensión de un concepto, lo que se denomina tipicidad. Se trata de que no todos los miembros en la extensión de un concepto son considerados igual de buenos representantes del concepto, sino que hay miembros más típicos de la clase que otros. Así, plátano es un caso típico de fruta, melón, intermedio, castaña es poco típico (aunque típico de la clase "fruto seco"), y tomate es borroso: hay razones para considerarlo una fruta, pero también para considerar que no lo es (como los calabacines, a pesar de ser una cucurbitácea como el melón).

No está tan clara la explicación de esta estructura gradual de pertenencia. Para Rosch, se debe a que nos representamos los conceptos como prototipos, esto es, como una combinación de características más o menos comunes a todos los miembros de la extensión. Este es el modo como Rosch concreta la idea de Wittgenstein de los parecidos de familia: no hay una esencia, expresada mediante la definición, del concepto. La intensión consiste en una serie de características, que pueden satisfacerse en diferentes grados, pero cuya importancia también puede ser variable, que determinan grados diferentes de pertenencia y, en algunos casos, borrosidad, vaguedad, dudas sobre la propiedad de aplicar el predicado a tal caso. La indeterminación de la extensión, por tanto, se debe a la multiplicidad de criterios de pertenencia de la intensión.

Un ejemplo analizado en detalle (Lakoff, 1987) es el de madre. El concepto de madre incluye una dimensión genética (la persona que aportó el óvulo), gestacional (quien tuvo el embarazo y parió), cuidadora (quien se encarga de atender las necesidades del bebé), jurídica (quien tiene o comparte la tutela). Quien satisface todas estas características sería una madre típica, pero es posible satisfacer solo algunas de ellas (madre de alquiler, madre biológica, madre adoptiva), e incluso casos en que esté indeterminado si puede aplicarse o no el concepto de madre (algunas madrastras que hacen honor a su fama de cuento), sobre todo teniendo en cuenta la restricción de que "madre no hay más que una".

Es objeto de discusión si es así como efectivamente se representan nuestros conceptos. En particular, para los categoriales (conceptos que podemos aplicar perceptivamente) resulta especialmente difícil acceder a las características que conforman el prototipo (o el modelo cognitivo idealizado, como también se ha llamado). Una alternativa teórica sugiere que los conceptos estarían representados en base a un ejemplar, un caso particular de referencia, junto a una métrica de la semejanza. Así, reconocer un caso como instancia del concepto dependería de juzgarlo suficientemente parecido, en los aspectos relevantes, al ejemplar de referencia. Si solo hemos conocido un finlandés, la idea es que aplicaremos el concepto de finlandés a cualquier que nos resulte suficientemente similar a nuestro amigo (en los aspectos relevantes). Desde el punto de vista explicativo, la diferencia clave es que este segundo enfoque no requiere la representación explícita de las características relevantes para pertenecer a la extensión, lo que resulta más coherente con el énfasis actual en los procesos implícitos en general, para los que no disponemos de evidencia introspectiva. Esta concepción, además, ha sido implementada con cierto éxito con modelos conexionistas, especialmente apropiados para reflejar la dependencia contextual de nuestros conceptos, la modulación de su valor semántico en función de con qué otros términos aparecen (dependencia contextual que caracteriza, como ya avanzamos, nuestros predicados típicamente graduales, como "alto", que permite entender la posibilidad de afirmar, tanto de Pedro, un chico de 10 años, que mide metro cuarenta, como de Gasol, con sus 2,18, que son altos: como si la extensión de "alto" no pudiera especificarse en términos de altura en centímetros). Pero más allá de si 
la representación es explícita o implícita, y del modo correcto de explicar la dependencia contextual de la semántica de nuestros predicados, ambos enfoques coinciden en el modo en que explican la aparición de casos borrosos: cuando un caso satisface una de las características pero no otra, o satisface en algún grado una característica poco típica pero escasamente una muy típica, etc. Como hemos visto en el caso de los sexos, se puede satisfacer el criterio genético pero no el hormonal, o a la inversa. O hacerlo en mayor o menor medida.

En algunos casos, además, conocidos como de percepción categorial (Harnad, 1987), encontramos no varias características combinadas, sino una segmentación de una única variable física en categorías psicológicas. Se trata de un mecanismo más básico de categorización de nuestra experiencia, que establece fronteras psicológicas categoriales donde no hay sino una magnitud física continua, pero que tiene el efecto de reducir la discriminabilidad de las diferencias dentro de la categoría y de acentuar las diferencias entre categorías. El caso de los fonemas, o de los colores, es representativo de este mecanismo cognitivo. No obstante, también en estos casos se plantean los mismos problemas de límites borrosos, de puntos de transición ambiguos, o cuya interpretación cognitiva depende del contexto. En tales casos, aunque solo haya un aspecto relevante, también se admiten grados, un continuo de grados de satisfacción del predicado.

Más allá de estas diferencias, lo importante de esta concepción contemporánea es el énfasis en los aspectos funcionales y pragmáticos de nuestro sistema conceptual, más que en la pretensión logicista-racionalista de encontrar en nuestros conceptos un reflejo de la estructura ontológica básica de la realidad. Así, desde esta concepción se insiste en el principio de economía cognitiva que impulsa nuestras abstracciones conceptuales, en el sentido de poder captar las contingencias robustas en nuestra experiencia (y por tanto, ignorar, abstraer los detalles de menos interés), en base a las cuales poder anticipar expectativas y predicciones, así como basarlas en nuestros patrones de interacción significativa con lo que nos rodea (en lugar de adoptar un punto de vista "divino", independiente de nuestra propia perspectiva como sujetos). Por supuesto, estos aspectos robustos de nuestra experiencia pueden necesitar de la transmisión social de conocimiento, del aprendizaje supervisado y la transmisión lingüística, más allá de la confrontación individual con el medio.

Estas consideraciones pragmáticas se han visto reforzadas por el desarrollo reciente de la perspectiva "incorporada" de la cognición, que defiende la necesidad de basar el contenido de nuestros conceptos en nuestras interacciones en último términos sensoriomotoras con nuestro entorno (físico o social). Sin duda el lenguaje proporciona un repertorio conceptual mucho más amplio, y distanciado de constancias perceptivas estrictas, pero la idea es que su significatividad debe basarse en tales interacciones, así como en los procesos sociales de transmisión, que son concebidos asimismo como interacciones en último término sensoriomotoras (Gomila, 2008). De ahí la robustez general del fenómeno de la dependencia del contexto: un niño puede ser alto si mide metro y medio a los ochos años, pero con esa altura no diríamos que un jugador de baloncesto es alto (caso de que existiera un caso así). En conjunto, desde este punto de vista, la vaguedad, la borrosidad, la indeterminación semántica en algunos casos deja de ser vista -como la ha visto siempre el logicista - como un defecto del que adolece nuestro lenguaje, que debe ser eliminado introduciendo mayor precisión y determinación semántica, sino como la consecuencia de nuestra arquitectura cognitiva y de la estructura continua y dinámica de la realidad ${ }^{3}$. Sin embargo, es preciso insistir en que la indeterminación semántica no depende de la borrosidad del predicado por sí mismo, sino del caso particular al que se aplica (aunque podría darse también el fenómeno del predicado borroso por inespecífico). Para volver con el ejemplo anterior: "alto" puede ser un concepto borroso, y dependiente del contexto, pero "Pau Gasol es alto" tiene un valor de verdad perfectamente determinado, en virtud de las características prototípicas de "alto" que Gasol ejemplifica en grado sumo. Que las fronteras sean borrosas no significa que no puedan ser cruzadas (que no sean útiles, que no capten diferencias o aspectos relevantes desde el punto de vista de la estructura causal de la realidad y su inteligibilidad). Al contrario, la posibilidad de una comunidad con prácticas conceptuales compartidas depende de la existencia de los casos paradigmáticos, prototípicos, centrales, de ejemplificación de un concepto.

\section{BORROSIDAD EN LOS CONCEPTOS MORALES $Y$ REALISMO MORAL}

Las ideas principales de la sección anterior se pueden resumir diciendo que nuestros predicados, por su estructura interna, se articulan en ejes graduales, de más o menos, en función de la tipicidad de los ejemplares, hasta el punto de la vaguedad o borrosidad de los casos límites. Y que la estrategia de combatir este fenómeno introduciendo nuevos conceptos, nuevas distinciones, para ser más precisos y eliminar tales casos límite, no puede tener éxito, porque solo se consigue reproducir la borrosidad para esos nuevos predicados, o en un segundo nivel. Por supuesto, esto no significa que debamos dar por buenos los predicados y distinciones heredados sin más, o que nunca tenga sentido revisar nuestros conceptos y su aplicación (puede ocurrir que descubramos inconsistencias 
en nuestras prácticas, u otros tipos de conflictos). Al contrario, parte del progreso moral puede tener que ver con entender mejor las diferencias relevantes y el modo de establecerlas. Se trata, no obstante, de oponernos al supuesto implícito de que en realidad no pueden darse casos borrosos, que los hechos deben estar perfectamente determinados, al margen de nuestra manera de concebirlos. En esta sección, trataré de mostrar que los conceptos morales también presentan el mismo fenómeno de tipicidad y estructura graduada, y por tanto, también deben darse casos borrosos, y argumentaré que tal situación no tiene por qué resultar incompatible con el realismo moral, del mismo modo que el fenómeno general de la tipicidad de nuestros conceptos categoriales no es incompatible con el realismo en general.

Los casos límite en la aplicación de los conceptos morales que hemos revisado anteriormente pueden surgir por las mismas razones que surgen en general. En particular, quisiera mencionar cuatro en concreto: puede darse el caso de que un concepto dependa de un criterio cuya satisfacción puede ser gradual; puede ocurrir que un concepto se articule en torno a más de un criterio, o concepción, que pueden divergir en su aplicación a algunos casos; también puede ocurrir, en casos de diversos criterios constitutivos, que esos criterios puedan satisfacerse también de manera gradual, tal como hemos visto en el caso del sexo (Shafer-Landau, 1995); y finalmente, es posible que una situación, un caso, permita conceptualizaciones diversas (en términos del concepto de libertad frente al de igualdad, por ejemplo). En cualquiera de estos casos, es esperable que puedan darse casos borrosos, o que podamos imaginar situaciones que constituyan casos límite de la aplicación de los conceptos involucrados.

Así, por ejemplo, el concepto de responsabilidad ilustra el caso de una propiedad gradual. Como se puede ser más o menos responsable, podemos imaginar - o encontrarnos en la vida real- un caso donde no resulta posible establecer si el protagonista de la situación era responsable de sus actos. "Honrado" podría ser un ejemplo de un concepto con varios criterios de aplicación, que pueden no converger en algún caso, sin que sea obvio establecer cuál de ellos debe tener el máximo peso. Así, consideramos honrado a quien no roba, y también a quien da a cada cual lo suyo, lo cual puede llevar a la indeterminación de la proposición "Robin Hood es honrado": se satisface un criterio pero el otro no. Incluso puede ocurrir que esos varios criterios presenten también grados de satisfacción, se pueda ser más o menos honrado, en función de cada criterio, lo que abre otra fuente de indeterminación. Por último, es posible que un caso genere un conflicto en torno a su mejor descripción en términos morales (en un caso de venganza puede no ser obvia la adjudicación de los papeles de vícti- ma y de verdugo). Es posible que los casos habituales no presenten tal borrosidad, pero como hemos visto antes, en la Bioética tales casos aparecen por nuevas posibilidades técnicas, o a través de situaciones hipotéticas, experimentos mentales, que ponen a prueba igualmente nuestras prácticas conceptuales.

Por supuesto, no es preciso para que la aplicación de un predicado sea gradual que se refiera a una magnitud continua. Por la raíz metafórica de nuestro sistema conceptual, el eje más-menos articula muchas de nuestras polaridades conceptuales, sin solución de continuidad, lo que nos lleva a distinguir grados, y casos típicos. Como se ha señalado anteriormente, esta gradualidad podría derivarse del modo en que se produce la transmisión social de las prácticas conceptuales: a través de la aplicación de los predicados a casos paradigmáticos (Wittgenstein, 1953), que constituyen el punto de referencia para la métrica de la semejanza: la semejanza siempre se da en grados, y depende del contexto. Por tanto, hay buenas razones para considerar los conceptos morales como iguales a los categoriales en lo que se refiere a su estructura gradual y, por tanto, a la posibilidad de que su aplicación en algunos casos resulte borrosa, dando lugar a proposiciones indeterminadas. Por lo menos no hay razones para tratar de modo diferente a este respecto a un tipo de afirmaciones o predicados frente al otro.

Como también hemos avanzado, la indeterminación semántica suele ser rechazada en razón del supuesto de que la realidad debe ser determinada. Este es el supuesto compartido tanto de los realistas como de los antirealistas. Los primeros, no obstante, la rechazan, mientras que los segundos son antirealistas precisamente en base a la existencia de tales proposiciones indeterminadas. No obstante, creo que puede aceptarse el realismo sin comprometerse con que todas las proposiciones deben tener un valor de verdad determinado. Si uno considera el caso de la descripción de la realidad, resulta cada vez más claro que ni siquiera en el plano de la descripción fundamental de la realidad vamos a encontrar satisfacción para esa aspiración racionalista. En esas teorías encontramos conceptos como el de campo, o espectro, o isótopo, que implican continuidad y cambio dinámico, por no hablar de la indeterminación de Heisenberg. En otros niveles de explicación ese mismo carácter continuo y dinámico de la realidad se acepta sin problema, a pesar de las dificultades que eso puede generar a la hora de describirla. Y ya ha quedado suficientemente claro el fenómeno de la tipicidad y borrosidad con respecto a nuestros conceptos de sentido común.

Mi sugerencia es que esa misma comprensión realista de nuestras prácticas en el discurso moral es igualmente posible, a pesar de que ocasionalmente nos encontremos con indeterminaciones, y con dilemas 
morales. El realismo está comprometido solamente con que la verdad de nuestras proposiciones morales no depende de nuestras propias decisiones, preferencias, actitudes, o prácticas, sino que depende de una realidad independiente (Dummett, 1982) ${ }^{4}$. El realismo hace justicia al modo en que utilizamos el lenguaje normativo (con pretensión de verdad, de corrección, de justificación), y, además, en la versión defendida aquí, es compatible con la indeterminación semántica en casos borrosos. Además, permite explicar la existencia de desacuerdos y de dilemas éticos, frente a quienes sostienen que constituyen la mejor prueba a favor del antirealismo. El realismo moral también nos lleva a tratar de mejorar nuestro conocimiento de la realidad, por su relevancia moral, sin tener que suponer que tal realidad esté perfectamente determinada. Lo cual hace recomendables los criterios de prudencia en los casos borrosos.
1 Por supuesto, no solo la Bioética, sino también otras disciplinas normativas como el derecho. Cf. Endicott (2007).

2 En Fausto-Sterling (2000) se recoge la nueva nomenclatura propuesta por la North American Task Force on Intersexuality, constituida por un grupo de pediatras, que de hecho, establecen fronteras distintas. Se distingue entre intersexuales tipo 1 (por influencias virilizantes), tipo 2 (por interrupción de proceso de virilización) y tipo 3 (por alteraciones del desarrollo de las gónadas). De nuevo, es una subclasificación de segundo orden, que presupone el carácter básico de la dicotomía masculinofemenino, y susceptible a nuevos casos límite. Además, el principio taxonómico es extraño en biología (las taxonomías son etiológicas o estructurales, no por el tipo de desarrollo).
3 Frente al fenómeno de la vaguedad e indeterminación semántica se plantean diversas alternativas en lógica filosófica y filosofía del lenguaje: atribuirla a nuestra limitación epistémica (Sorensen, 1988), a la inadecuación ontológica de nuestros conceptos de sentido común (Heller, 1991), o a su insuficiente precisión, para que lo se propone hacer preciso el ámbito de la indeterminación: es la estrategia supervaluacionista de, por ejemplo, Fine (1975); para una crítica de la viabilidad de esta estrategia, vd. Fodor y Lepore (1996). En cualquier caso, todos estos planteamientos suelen compartir el supuesto de que la propia realidad tiene que ser determinada, de que los casos límite y la indeterminación semántica son inaceptables en principio. Como si la vaguedad fuera semejante a la ambigüedad: si de la existencia de predicados ambiguos no inferimos la existencia de objetos ambiguos, de la existencia de predicados vagos no deberíamos in-

Evans, G. (1975). "Can there be vague objects?". Analysis, 38, p. 208.

Fausto-Sterling, A. (1993). "The five sexes". The Sciences, marzo/abril.

Fausto-Sterling, A. (2000). Sexing the body. New York: Basic Books.

Fine, K. (1975). "Vagueness, truth and logic". Synthèse, 30, pp. 265-300.

Fodor, J. \& Lepore, E. (1996). "What cannot be evaluated cannot be evaluated, and it cannot be superevaluated either". Journal of Philosophy, 93, pp. 516-535.

Gomila, A. (2008). "Mending or abandoning cognitivism?". En A. Glenberg, M. de Vega y A. Glaesser (eds.). Symbols, ferir la existencia de objetos vagos (es el argumento clásico de Rusell, 1923; vd. también Evans, 1975). El único enfoque que parte de reconocer la posible borrosidad de las propiedades es el de la "lógica fuzzy" o borrosa (Zadeh, 1982), aunque lo hace al precio de reinterpretar la indeterminación semántica en términos de probabilidades, con lo que se confunde indeterminación con incertidumbre. Una estrategia en esta línea, aunque influida por la lógica paraconsistente, es la que sigue Ausín (2006).

4 Obviamente, la realidad moral es una realidad vinculada a la experiencia humana, y nuestro conocimiento de ella está íntimamente ligado a nuestros conceptos morales, a nuestra autocomprensión como agentes morales. Pero eso no impide que podamos estar equivocados o cometer errores morales, lo cual es difícil de explicar desde un planteamiento antirealista.

Ausín, T. (2006). Entre la lógica y el derecho: paradojas y conflictos normativos. Ma-

thers, P. F. (2005). Consciousness Oxford: Oxford University Press.

Dummett, M. (1982). "Realism". Synthèse, 52, pp. 55-112.

Endicott, T. (2007). La vaguedad en el dere cho. Madrid: Dykinson.

Embodiment and Meaning, Oxford: Oxford University Press, pp. 799-834.

Harnad, S. (1987). Categorical perception: the groundwork of cognition. New York: Cambridge University Press.

Kamp, H. \& Partee, B. (1995). "Prototype theory and Compositionalist". Cognition, 57, pp. 129-191.

Heller, M. (1975). "Vagueness and the standard ontology". Nous, 22, pp. 325-366.

Lakoff, G. (1987). Women, fire, dangerous things. What categories reveal about the mind. Chicago: University of Chicago Press. 
Rabossi, E. (2002). "Las falacias filosóficas y el estigma del naturalismo". En D. Pérez (comp.). Los caminos del naturalismo. Mente, conocimiento y moral. Buenos Aires: Eudeba, pp. 17-46.

Rosch, E. \& Mervis, C. (1975). "Family resemblance: studies in the internal structure of categories". Cognitive Psychology, 7, pp. 573-605.

Russell, B. (1923). "Vagueness". Australasian Journal of Philosophy, 1, pp. 84-92.
Shafer-Landau, R. (1995). "Vagueness, borderline case and moral realism". American Philosophical Quarterly, 32, pp. 83-95.

Sinnot-Armstrong, W. (1988). Moral dilemmas. Oxford: B. Blackwell.

Sorensen, R. A. (1988). Blindspots. Oxford: Clarendon Press.

Sorensen, R.A. (1991). "Moral dilemmas, thoughts experiments and conflict va- gueness". Philosophical Studies, 63, pp. 291-308.

Wittgenstein, L. (1953). Philosophical Investigations. Oxford: Blackwell Publishing.

Zadeh, L. (1982). "A note on prototype theory and fuzzy sets". Cognition, 12, pp. 291-298. 